\title{
Extensions of the Cugiani-Mahler theorem
}

\author{
YANN BUGEAUD
}

\begin{abstract}
In 1955, Roth established that if $\xi$ is an irrational number such that there are a positive real number $\varepsilon$ and infinitely many rational numbers $p / q$ with $q \geq 1$ and $|\xi-p / q|<q^{-2-\varepsilon}$, then $\xi$ is transcendental. A few years later, Cugiani obtained the same conclusion with $\varepsilon$ replaced by a function $q \mapsto \varepsilon(q)$ that decreases very slowly to zero, provided that the sequence of rational solutions to $|\xi-p / q|<q^{-2-\varepsilon(q)}$ is sufficiently dense, in a suitable sense. We give an alternative, and much simpler, proof of Cugiani's Theorem and extend it to simultaneous approximation.
\end{abstract}

Mathematics Subject Classification (2000): 11J68.

\section{Introduction}

In 1955, Roth [26] established that, like almost all real numbers (throughout the present paper, 'almost all' refers to the Lebesgue measure), an algebraic irrational number cannot be approximated by rationals at an order greater than 2 .

Theorem 1.1 (Roth, 1955). Let $\xi$ be an irrational, algebraic real number. Let $\varepsilon$ be a positive real number. Then there are only finitely many rational numbers $p / q$ with $q \geq 1$ such that

$$
\left|\xi-\frac{p}{q}\right|<\frac{1}{q^{2+\varepsilon}} .
$$

As pointed out by Mahler in Appendix B of [21], Roth's theorem suggests the following problem.

Problem 1.2. Let $\xi$ be an irrational, algebraic real number. To find a positive function $q \mapsto \varepsilon(q)$ of the integral variable $q$, with the property

$$
\lim _{q \rightarrow+\infty} \varepsilon(q)=0
$$

such that there are at most finitely many distinct rational numbers $p / q$ with positive denominator for which

$$
\left|\xi-\frac{p}{q}\right|<\frac{1}{q^{2+\varepsilon(q)}} .
$$

Received April 23, 2007; accepted in revised form September 7, 2007. 
If one believes that real algebraic numbers do behave like almost all real numbers as far as rational approximation is concerned, then Problem 1.2 should have a positive answer with the function $\varepsilon$ given by

$$
q \longmapsto(1+\eta) \frac{\log \log q}{\log q},
$$

for an arbitrary positive number $\eta$.

As written by Mahler, 'the method of Roth does not seem strong enough for solving this problem'; however, a weaker result was found by Cugiani [10] in 1958.

Theorem 1.3 (Cugiani, 1958). Let $\xi$ be a real algebraic number of degree $d$. For an integer $q \geq 16$, set

$$
\varepsilon(q)=\frac{9 d}{(\log \log \log q)^{1 / 2}} .
$$

Let $\left(p_{j} / q_{j}\right)_{j \geq 1}$ be the sequence of reduced rational solutions of

$$
\left|\xi-\frac{p}{q}\right|<\frac{1}{q^{2+\varepsilon(q)}}
$$

ordered such that $16 \leq q_{1}<q_{2}<\ldots$ Then either the sequence $\left(p_{j} / q_{j}\right)_{j \geq 1}$ is finite, or

$$
\limsup _{j \rightarrow+\infty} \frac{\log q_{j+1}}{\log q_{j}}=+\infty .
$$

The above theorem was subsequently generalized by Cugiani $[11,12]$ and Mahler [21] to include non-Archimedean valuations, and is now referred to as the CugianiMahler theorem. Its extension to approximation by elements from a given number field was worked out by Rodriquez [24,25].

A further improvement was obtained in 1988 by Bombieri and van der Poorten [8], who used the Dyson lemma of Esnault and Viehweg in place of the Roth lemma and were able to derive the Cugiani-Mahler theorem with a function $\varepsilon$ that decreases to zero faster than in (1.3).

Theorem 1.4 (Bombieri and van der Poorten, 1988). Let $\xi$ be a real algebraic number of degree $d$. For an integer $q \geq 4$, set

$$
\varepsilon(q)=7(\log 4 d)^{1 / 2}\left(\frac{\log \log \log 4 q}{\log \log 4 q}\right)^{1 / 4} .
$$

Let $\left(p_{j} / q_{j}\right)_{j \geq 1}$ be the sequence of reduced rational solutions of

$$
\left|\xi-\frac{p}{q}\right|<\frac{1}{q^{2+\varepsilon(q)}},
$$

ordered such that $4 \leq q_{1}<q_{2}<\ldots$ Then either the sequence $\left(p_{j} / q_{j}\right)_{j \geq 1}$ is finite or

$$
\limsup _{j \rightarrow+\infty} \frac{\log q_{j+1}}{\log q_{j}}=+\infty .
$$


As far as we are aware, the above theorem provides currently the best contribution towards a resolution of Problem 1.2.

At the end of the 60's, multidimensional extensions of Roth's theorem were established by W. M. Schmidt [27-29]. We extract the following statements from Chapter VI of [31]. Given a real number $x$, we denote by $\|x\|$ the distance from $x$ to the nearest integer. Given an algebraic number $\alpha$, we denote by $H(\alpha)$ its height, that is, the maximal of the absolute values of the coefficients of its minimal defining polynomial over the ring of integers.

Theorem 1.5 (Schmidt, 1970). Let $n$ be a positive integer. Let $\xi_{1}, \ldots, \xi_{n}$ be algebraic real numbers such that $1, \xi_{1}, \ldots, \xi_{n}$ are linearly independent over the rationals. Let $\varepsilon$ be a positive real number. Then, there exist only finitely many positive integers $q$ such that

$$
q \cdot\left\|q \xi_{1}\right\| \cdots\left\|q \xi_{n}\right\|<q^{-\varepsilon} .
$$

Theorem 1.6 (Schmidt, 1970). Let $n$ be a positive integer. Let $\xi$ be a real algebraic number of degree greater than $n$. Let $\varepsilon$ be a positive real number. Then, there exist only finitely many algebraic numbers $\alpha$ of degree at most $n$ such that

$$
|\xi-\alpha|<H(\alpha)^{-n-1-\varepsilon} .
$$

Schmidt [30] established a general result in 1972, commonly referred to as the Schmidt Subspace theorem, and from which the above two theorems follow. The quantitative version of the Schmidt Subspace theorem is quoted as Theorem ES in Section 4. No multidimensional analogue of the Cugiani-Mahler theorem has been published yet. Our main purpose is precisely to establish such a statement.

Let us now describe the structure of the present work. We begin with giving a (presumably) new proof of a stronger version of the above quoted theorem of Cugiani: we show that it easily follows from an upper estimate for the number of solutions to (1.1). Related results are stated in Section 2 and proved in Section 5.

This new approach allows us to establish multidimensional extensions of the Cugiani-Mahler theorem, by means of a quantitative version of the Schmidt Subspace theorem. The corresponding statements are gathered in Section 3 and proved in Section 6.

An application of the Cugiani-Mahler theorem to fractional parts of powers of rational numbers is discussed in Section 7. The paper ends with several remarks gathered in Section 8.

\section{Small variations around the Cugiani-Mahler theorem}

The single paper on the Cugiani-Mahler theorem that we do not have quoted in Section 1 was published by Mignotte [22]. It contains an improvement upon the gap condition (1.4). Mignotte's result can be formulated as follows. 
Theorem 2.1 (Mignotte, 1972). Let $\xi$ be a real algebraic number of degree $d$. Let $\alpha$ be real, $0<\alpha<6$. For an integer $q \geq 16$, set

$$
\varepsilon(q)=\left(\frac{16 \log (d+2) \log 2}{\alpha \log \log \log q}\right)^{1 / 2} .
$$

Let $\left(p_{j} / q_{j}\right)_{j \geq 1}$ be the sequence of reduced rational solutions of

$$
\left|\xi-\frac{p}{q}\right|<\frac{1}{q^{2+\varepsilon(q)}},
$$

ordered such that $16 \leq q_{1}<q_{2}<\ldots$ Then either the sequence $\left(p_{j} / q_{j}\right)_{j \geq 1}$ is finite or, for any real number $\rho$ with $1<\rho<2^{(6-\alpha) / \alpha}$, we have

$$
\limsup _{j \rightarrow+\infty} \frac{\log q_{j+1}}{\left(\log q_{j}\right)^{\rho}}=+\infty .
$$

Bombieri and van der Poorten noted at the end of [8] that, if one wishes to get a gap condition as in (2.1), their method only yields a result of the quality of Mignotte's.

The main result of this section is in the same spirit as the Cugiani-Mahler theorem. It is an easy consequence of an estimate of Evertse [14] for the number of solutions to (1.1).

Theorem 2.2. Let $\xi$ be a real algebraic number of degree $d$. Let $\varepsilon$ be a nonincreasing function defined over the set of positive integers and tending to 0 at infinity. Assume that there is an infinite sequence $\left(p_{j} / q_{j}\right)_{j \geq 1}$ of reduced rational numbers such that $1 \leq q_{1}<q_{2}<\ldots$ and

$$
\left|\xi-\frac{p_{j}}{q_{j}}\right|<\frac{1}{q_{j}^{2+\varepsilon\left(q_{j}\right)}}, \quad \text { for } j \geq 1 .
$$

Then, there exist a constant $c_{1}(d)$, depending only on the degree $d$ of $\xi$, and a constant $c_{2}(\xi)$, depending only on $\xi$, such that

$$
\varepsilon\left(q_{j}\right) \leq c_{1}(d) j^{-1 / 3}(\log j)^{2 / 3}, \quad \text { for } j \geq c_{2}(\xi) .
$$

Theorem 2.2 asserts that if (1.2) has infinitely many solutions, then there is a big gap between any two of these solutions, and the size of these gaps increases when the function $\varepsilon$ decreases slowly to zero.

We display an immediate corollary of Theorem 2.2. For a positive integer $m$, we denote by $\exp _{m}$ the $m$ th iterate of the exponential function and by $\log _{m}$ the function that coincides with the $m$ th iterate of the logarithm function on $\left[\exp _{m} 1,+\infty\right)$ and that takes the value 1 on $\left(-\infty, \exp _{m} 1\right]$. We also adopt the convention that $\log _{0}$ coincides with the identity function. 
Corollary 2.3. Let $m$ be a positive integer and set

$$
\varepsilon(q)=\left(\log _{m+1} q\right)^{-1 / 3}\left(\log _{m+2} q\right), \quad \text { for } q \geq 1 .
$$

Let $\xi$ be a real algebraic number and let $\left(p_{j} / q_{j}\right)_{j \geq 1}$ be the sequence of reduced rational solutions of

$$
\left|\xi-\frac{p}{q}\right|<\frac{1}{q^{2+\varepsilon(q)}}
$$

ordered such that $1 \leq q_{1}<q_{2}<\ldots$ If the sequence $\left(p_{j} / q_{j}\right)_{j \geq 1}$ is infinite, then

$$
\lim _{j \rightarrow+\infty} \frac{\log _{m+1} q_{j}}{j}=+\infty
$$

and, consequently,

$$
\limsup _{j \rightarrow+\infty} \frac{\log _{m} q_{j+1}}{\log _{m} q_{j}}=+\infty
$$

Taking $m=1$ in Corollary 2.3, we get a (slight) improvement of the theorem of Bombieri and van der Poorten. Choosing $m=2$ in Corollary 2.3, we get a result comparable to Mignotte's theorem.

All the previous proofs of the Cugiani-Mahler theorem were obtained by a suitable modification of the proof of Roth's theorem (see also [33, Exercise 7.5.5]), and thus are much more technical than the proof of Theorem 2.2. Actually, the complications of the present proof are hidden in the proof of Evertse's estimate for the number of solutions to (1.1), recalled in Section 4.

It is of interest to note that if we insert the upper bound established in 1955 by Davenport and Roth [13] for the number of solutions to (1.1) in place of Evertse's estimate in the proof of Theorem 2.2, then we get a very short proof of the above theorem of Cugiani, with the function $\varepsilon$ given in (1.3) replaced by the function

$$
q \mapsto(\log \log \log q)^{-1 / 2}(\log \log \log \log q), \quad\left(q \geq 10^{8}\right) .
$$

The fact that the sequence $\left(q_{j}\right)_{j \geq 1}$ increases as rapidly as given by (2.2) has not been pointed out in the earlier works on the Cugiani-Mahler theorem. This is much stronger than (2.3), which says only that there are infinitely many large gaps in the sequence $\left(q_{j}\right)_{j \geq 1}$. This improvement follows from a simple gap principle saying that $\left(q_{j}\right)_{j \geq 1}$ increases at least exponentially. Unfortunately, we were unable to establish analogues of (2.2) for our Theorems 2.4, 3.1 and 3.2 below. This is due to the lack of a sufficiently strong gap principle.

The proof of Theorem 2.2 is very flexible and can be easily adapted to include non-Archimedean valuations, as in a result of Ridout [23]; we simply have to replace the use of Evertse's estimate by a result of Locher [19] to get the following theorem.

For a prime number $\ell$ and a non-zero rational number $x$, we set $|x|_{\ell}:=\ell^{-u}$, where $u$ is the exponent of $\ell$ in the prime decomposition of $x$. Furthermore, we set $|0|_{\ell}=0$. 
Theorem 2.4. Let $S$ be a finite set of prime numbers, and denote by s its cardinality. Let $\eta$ be a positive real number. Let $m$ be a positive integer and set

$$
\varepsilon(q)=\left(\log _{m+1} q\right)^{-1 /(s+5+\eta)}, \quad \text { for } q \geq 1 .
$$

Let $\xi$ be a real algebraic number and let $\left(p_{j} / q_{j}\right)_{j \geq 1}$ be the sequence of reduced rational solutions of

$$
\min \left\{1,\left|\xi-\frac{p}{q}\right|\right\} \cdot \prod_{\ell \in S}|p q|_{\ell}<\frac{1}{q^{2+\varepsilon(q)}},
$$

ordered such that $1 \leq q_{1}<q_{2}<\ldots$ Then either the sequence $\left(p_{j} / q_{j}\right)_{j \geq 1}$ is finite or

$$
\limsup _{j \rightarrow+\infty} \frac{\log _{m} q_{j+1}}{\log _{m} q_{j}}=+\infty .
$$

We point out that the function $\varepsilon$ occurring in Theorem 2.4 does depend on the set of primes $S$, while this is not the case in Theorem 6.5.10 from Chapter 6 of the monograph of Bombieri and Gubler [7]. The latter is given without proof, and we were unable to find the key ingredient used by Bombieri and Gubler to remove the dependence on the cardinality of $S$.

Versions of Theorem 2.4 in which (2.4) is replaced by a system of inequalities were proved by Mahler [21] and Mignotte [22]. It does not seem to be easy to deduce Theorem 2.4 from their results: the difficulty lies in the fact that we have a product in the left-hand side of (2.4).

Theorem 2.4 can be used to give new explicit examples of transcendental numbers, by proceeding exactly as Mahler did, see [21, Theorem 3, page 178] and [22, Théorème 3]. Theorem 2.4 also implies new results on the distribution modulo one of the sequence of integral powers of a rational number greater than one: see [21, Theorem 2, page 176] and [22, Théorème 2]. We discuss more closely the latter application in Section 7. As for the former one, which actually do not need the full power of Theorem 2.4, the reader is referred to Section 9 of [9].

Finally, we stress that Theorems 2.2 and 2.4 can be extended to approximation by elements of a given number field, a setting considered in [8].

\section{Multidimensional extensions of the Cugiani-Mahler theorem}

Our new proof of the Cugiani-Mahler theorem extends well to multidimensional approximation and allows us to generalize it in several directions.

For a positive real number $\eta$ and for irrational numbers $\xi_{1}, \ldots, \xi_{n}$, we say that the positive integer $q$ corresponds to a primitive solution of

$$
q \cdot\left\|q \xi_{1}\right\| \cdots\left\|q \xi_{n}\right\|<\eta
$$

if, denoting by $p_{j}$ the nearest integer to $q \xi_{j}$ for $j=1, \ldots, n$, the $(n+1)$-tuple $\left(q, p_{1}, \ldots, p_{n}\right)$ is primitive, that is, if the greatest common divisor of $q, p_{1}, \ldots, p_{n}$ 
is equal to 1 . Observe that, for $n=1$, the positive integer $q$ corresponds to a primitive solution of $q \cdot|q \xi-p|<\eta$ if the rational $p / q$ is written in reduced form.

Theorem 3.1. Let $n$ be a positive integer and $\xi_{1}, \ldots, \xi_{n}$ be real algebraic numbers such that $1, \xi_{1}, \ldots, \xi_{n}$ are linearly independent over the rationals. Let $\varepsilon: \mathbb{Z}_{\geq 1} \rightarrow$ $\mathbb{R}_{>0}$ be a non-increasing function satisfying

$$
\lim _{q \rightarrow+\infty} \frac{\varepsilon(q)}{(\log \log q)^{-1 /(2 n+6)}}=+\infty .
$$

Let $\left(q_{j}\right)_{j \geq 1}$ be the sequence of positive integers corresponding to primitive solutions of

$$
q \cdot\left\|q \xi_{1}\right\| \cdots\left\|q \xi_{n}\right\|<q^{-\varepsilon(q)},
$$

ordered such that $1 \leq q_{1}<q_{2}<\ldots$ If this sequence is infinite, then

$$
\limsup _{j \rightarrow+\infty} \frac{\log q_{j+1}}{\log q_{j}}=+\infty
$$

Theorem 3.1 provides a first step towards a (small) improvement on the first result of Schmidt quoted in the Introduction.

The second statement of Schmidt quoted in the Introduction is an easy consequence of a deep result from [29] asserting that, if $\xi_{1}, \ldots, \xi_{n}$ are real algebraic numbers such that $1, \xi_{1}, \ldots, \xi_{n}$ are linearly independent over the rationals, then, for any positive $\varepsilon$, there are only finitely many non-zero integer $(n+1)$-tuples $\left(p_{0}, \ldots, p_{n}\right)$ with

$$
\left|p_{0}+p_{1} \xi_{1}+\ldots+p_{n} \xi_{n}\right|<\left(\max \left\{\left|p_{0}\right|,\left|p_{1}\right|, \ldots,\left|p_{n}\right|\right\}\right)^{-n-\varepsilon} .
$$

Our next statement is an extension of the Cugiani-Mahler theorem to this setting.

Theorem 3.2. Let $n$ be a positive integer and $\xi_{1}, \ldots, \xi_{n}$ be real algebraic numbers such that $1, \xi_{1}, \ldots, \xi_{n}$ are linearly independent over the rationals. Let $\varepsilon: \mathbb{Z}_{\geq 1} \rightarrow$ $\mathbb{R}_{>0}$ be a non-increasing function satisfying

$$
\lim _{H \rightarrow+\infty} \frac{\varepsilon(H)}{(\log \log H)^{-1 /(2 n+6)}}=+\infty .
$$

Let $\left(p_{0, j}, p_{1, j}, \ldots, p_{n, j}\right)_{j \geq 1}$ be the sequence of primitive solutions of

$$
\left|p_{0}+p_{1} \xi_{1}+\ldots+p_{n} \xi_{n}\right|<H^{-n-\varepsilon(H)}, \quad H=\max \left\{\left|p_{0}\right|, \ldots,\left|p_{n}\right|\right\},
$$

ordered such that $1 \leq H_{1} \leq H_{2} \leq \ldots$, where $H_{j}=\max \left\{\left|p_{0, j}\right|, \ldots,\left|p_{n, j}\right|\right\}$ for $j \geq 1$. If this sequence is infinite, then

$$
\limsup _{j \rightarrow+\infty} \frac{\log H_{j+1}}{\log H_{j}}=+\infty .
$$


We display two corollaries of Theorem 3.2.

For an integer polynomial $P(X)$, we denote by $H(P)$ its height, that is, the maximum of the absolute values of its coefficients. Recall that, if $\alpha$ is an algebraic number, then its height, denoted by $H(\alpha)$, is the height of its minimal defining polynomial over the ring of integers.

Corollary 3.3. Let $n$ be a positive integer and $\xi$ be a real algebraic number of degree greater than $n$. Let $\varepsilon: \mathbb{Z}_{\geq 1} \rightarrow \mathbb{R}_{>0}$ be a non-increasing function satisfying

$$
\lim _{H \rightarrow+\infty} \frac{\varepsilon(H)}{(\log \log H)^{-1 /(2 n+6)}}=+\infty .
$$

Let $\left(P_{j}(X)\right)_{j \geq 1}$ be the sequence of distinct primitive, integer polynomials of degree at most $n$ that satisfy

$$
|P(\xi)|<H(P)^{-n-\varepsilon(H(P))},
$$

ordered such that $1 \leq H\left(P_{1}\right) \leq H\left(P_{2}\right) \leq \ldots$ If this sequence is infinite, then

$$
\limsup _{j \rightarrow+\infty} \frac{\log H\left(P_{j+1}\right)}{\log H\left(P_{j}\right)}=+\infty .
$$

Next corollary provides a first step towards a (small) improvement on the second result of Schmidt quoted in the Introduction.

Corollary 3.4. Let $n$ be a positive integer and $\xi$ be a real algebraic number of degree greater than $n$. Let $\varepsilon: \mathbb{Z}_{\geq 1} \rightarrow \mathbb{R}_{>0}$ be a non-increasing function satisfying

$$
\lim _{H \rightarrow+\infty} \frac{\varepsilon(H)}{(\log \log H)^{-1 /(2 n+6)}}=+\infty .
$$

Let $\left(\alpha_{j}\right)_{j \geq 1}$ be the sequence of distinct algebraic numbers of degree at most $n$ that satisfy

$$
|\xi-\alpha|<H(\alpha)^{-n-1-\varepsilon(H(\alpha))},
$$

ordered such that $1 \leq H\left(\alpha_{1}\right) \leq H\left(\alpha_{2}\right) \leq$.. If this sequence is infinite, then

$$
\limsup _{j \rightarrow+\infty} \frac{\log H\left(\alpha_{j+1}\right)}{\log H\left(\alpha_{j}\right)}=+\infty .
$$

If one believes that algebraic numbers behave like almost all numbers as far as approximation by algebraic numbers of larger degree is concerned, then results of Beresnevich [5] and Bernik [6] imply that inequalities (3.5) and (3.7) with a nonincreasing function $\varepsilon$ satisfying

$$
\lim _{H \rightarrow+\infty} \frac{\varepsilon(H)}{(\log H)^{-1}(\log \log H)}>1
$$

should have only finitely many solutions. 


\section{Auxiliary results}

We begin this section by stating a version of the Liouville inequality that follows from [34, Proposition 3.14].

Lemma 4.1. Let $n$ be a positive integer and $\xi_{1}, \ldots, \xi_{n}$ be real algebraic numbers in a number field of degree D. If $x_{1}, \ldots, x_{n}$ are rational integers such that $x_{1} \xi_{1}+$ $\ldots+x_{n} \xi_{n}$ is non-zero, then there exists a positive real number $H$, depending only on $n, \xi_{1}, \ldots, \xi_{n}$, such that

$$
\left|x_{1} \xi_{1}+\ldots+x_{n} \xi_{n}\right| \geq(X H)^{-D},
$$

where we have set $X:=\max \left\{\left|x_{1}\right|, \ldots,\left|x_{n}\right|\right\}$.

The first explicit upper bound for the number of solutions to (1.1) was established by Davenport and Roth [13] in 1955. It has been subsequently refined by several authors, and the current best estimate has been obtained by Evertse [14]. We gather in the same statement his result and an estimate of Locher [19].

Theorem EL. Let $S$ be a finite set of prime numbers and denote by sits cardinality. Let $\xi$ be an algebraic number of degree $d$ with $0<\xi<1$. Let $\varepsilon$ be a positive real number with $\varepsilon<1 / 5$. The inequality

$$
\min \left\{1,\left|\xi-\frac{p}{q}\right|\right\} \cdot \prod_{\ell \in S}|p q|_{\ell}<\frac{1}{q^{2+\varepsilon}}
$$

has at most

$$
\mathcal{N}_{1}(\xi, \varepsilon):=e^{8 s+26} \varepsilon^{-s-5} \log (6 d) \cdot \log \left(\varepsilon^{-1} \log (6 d)\right)
$$

reduced rational solutions $p / q$ with $q \geq \max \left\{4^{4 / \varepsilon}, \sqrt{d+1} H(\xi)\right\}$. Moreover, if $S$ is empty, then (4.1) can be replaced by

$$
\mathcal{N}_{2}(\xi, \varepsilon):=2 \cdot 10^{7} \varepsilon^{-3}\left(\log \varepsilon^{-1}\right)^{2}(\log 4 d)(\log \log 4 d) .
$$

Proof. Theorem 2 from [19] gives an upper bound for the number of reduced rational solutions to

$$
\min \left\{1,\left|\xi-\frac{p}{q}\right|\right\} \cdot \prod_{\ell \in S_{1}}|p|_{\ell} \cdot \prod_{\ell \in S_{2}}|q|_{\ell}<\frac{1}{q^{2+\varepsilon}},
$$

where $S_{1}$ and $S_{2}$ are disjoint finite sets of prime numbers whose union is equal to $S$. Since we have $2^{s}$ choices for the pair $\left(S_{1}, S_{2}\right)$, the bound (4.1) then follows.

Furthermore, the estimate established at the end of Section 6 from [14] implies (4.2). 
At present, we do not have any general upper bound for the number of solutions to the inequalities (1.5) and (1.6). However, if we define in (1.5) the integers $p_{1}, \ldots, p_{n}$ by $\| q \xi_{i}||=\left|q \xi_{i}-p_{i}\right|$ for $i=1, \ldots, n$, an upper estimate for the number of proper rational subspaces of $\mathbb{Q}^{n+1}$ in which the $(n+1)$-tuples $\left(q, p_{1}, \ldots, p_{n}\right)$ associated with the solutions of (1.5) are contained was obtained in a deep work of Schmidt [32], who established a quantitative version of his Subspace theorem. We state below a general result of Evertse and Schlickewei [15]. We refer to [15] for the definition of the height of a linear form, a notion that will not be used in the present paper.

Theorem ES. Let $m \geq 2$ be an integer. Let $L_{1}, \ldots, L_{m}$ be a linearly independent system of linear forms with real algebraic coefficients, and let $D$ be the degree of the number field generated by their coefficients. We assume that

$$
\operatorname{det}\left(L_{1}, \ldots, L_{m}\right)= \pm 1 .
$$

Let $H$ be an upper bound for the heights of the linear forms $L_{1}, \ldots, L_{m}$. Let $\varepsilon$ be a real number satisfying $0<\varepsilon<1$. Then, the set of primitive solutions $\mathbf{x}=$ $\left(x_{1}, \ldots, x_{m}\right)$ in $\mathbb{Q}^{m}$ to the inequality

$$
\prod_{i=1}^{m}\left|L_{i}(\mathbf{x})\right|<H(\mathbf{x})^{-\varepsilon}
$$

with

$$
\max \left\{\left|x_{1}\right|, \ldots,\left|x_{m}\right|\right\}>\max \left\{m^{4 m / \varepsilon}, H\right\}
$$

lies in the union of at most

$$
(3 m)^{2 m} 2^{3(m+9)^{2}} \varepsilon^{-2 m-4}(\log 4 D)(\log \log 4 D)
$$

proper subspaces of $\mathbb{Q}^{m}$.

Proof. This follows from Theorem 3.1 of Evertse and Schlickewei [15].

\section{Proofs of Theorems 2.2 and 2.4 and of Corollary 2.3}

Proof of Theorem 2.2. Keep the notation of Theorem 2.2. An almost forgotten result of Fatou [16] (see Grace [18] for a complete proof) asserts that if the rational number $a / b$ satisfies $|\xi-a / b|<1 / b^{2}$, then there exists an integer $n$ such that

$$
\frac{a}{b} \text { belongs to }\left\{\frac{r_{n}}{s_{n}}, \frac{r_{n+1}+r_{n}}{s_{n+1}+s_{n}}, \frac{r_{n+2}-r_{n+1}}{s_{n+2}-s_{n+1}}\right\},
$$

where $\left(r_{n} / s_{n}\right)_{n \geq 1}$ is the sequence of convergents to $\xi$. Furthermore, it is well known that

$$
s_{n} \geq(\sqrt{2})^{n-1}, \quad \text { for } n \geq 1 .
$$


Since by assumption $\left|\xi-p_{j} / q_{j}\right|<1 / q_{j}^{2}$, the above observations imply that

$$
q_{j} \geq 1.1^{j}, \quad \text { for } j \geq 2 .
$$

Let $j$ be a positive integer, and denote by $u$ the integer part of $j / 2$. We take $j$ sufficiently large in order that $q_{u} \geq \sqrt{d+1} H(\xi)$. We can assume that $\varepsilon\left(q_{j}\right) \geq$ $100 j^{-1}$, for otherwise the conclusion of the theorem clearly holds.

Consequently,

$$
q_{u} \geq 1.03^{j} \geq 4^{2 / \varepsilon\left(q_{j}\right)},
$$

and we infer from the last assertion of Theorem EL that

$$
\frac{j}{2} \leq j-u \leq 2 \cdot 10^{7} \varepsilon\left(q_{j}\right)^{-3}\left(\log \varepsilon\left(q_{j}\right)^{-1}\right)^{2}(\log 4 d)(\log \log 4 d) .
$$

This gives the desired result.

Proof of Corollary 2.3. Let $\xi$ be as in the statement of the corollary. Throughout this proof, the constants implied by $\ll$ and $\gg$ may depend on $\xi$, but are independent of $j$. Assuming that the sequence $\left(p_{j} / q_{j}\right)_{j \geq 1}$ is infinite, it follows from Theorem 2.2 that

$$
\left(\log _{m+1} q_{j}\right)^{-1 / 3}\left(\log _{m+2} q_{j}\right) \ll j^{-1 / 3}(\log j)^{2 / 3}, \quad \text { for } j \geq 1 .
$$

Thus, we have

$$
\left(\log _{m+1} q_{j}\right)\left(\log _{m+2} q_{j}\right)^{-3} \gg j(\log j)^{-2}, \quad \text { for } j \geq 1,
$$

and

$$
\lim _{j \rightarrow+\infty} \frac{\log _{m+1} q_{j}}{j}=+\infty
$$

If

$$
\log _{m} q_{j+1} \ll \log _{m} q_{j}, \quad \text { for } j \geq 1,
$$

then an easy induction shows that

$$
\log _{m+1} q_{j} \ll j, \quad \text { for } j \geq 1,
$$

a contradiction with (5.1). This establishes the corollary. 
Proof of Theorem 2.4. Keep the notation of the theorem. Without any restriction, we may assume that $0<\xi<1$. Assume that there is an absolute constant $C$ and an infinite sequence $\left(p_{j} / q_{j}\right)_{j \geq 1}$ of reduced rational numbers with $q_{j} \geq 1$ that satisfy (2.4) and

$$
2 \log _{m} q_{j} \leq \log _{m} q_{j+1} \leq C \log _{m} q_{j}, \quad \text { for } j \geq 1 .
$$

Let $N$ be an integer and set $\varepsilon=\varepsilon\left(q_{N}\right)$. Then, the inequality

$$
\min \left\{1,\left|\xi-\frac{p}{q}\right|\right\} \cdot \prod_{\ell \in S}|p q|_{\ell}<\frac{1}{q^{2+\varepsilon}}
$$

has at least $N$ solutions and, provided that $N$ is large enough, at least $N / 2$ among them have a denominator greater than $\max \left\{4^{4 / \varepsilon}, \sqrt{d+1} H(\xi)\right\}$, where $d$ is the degree of $\xi$. The constants implied by $\ll$ occurring below depend at most on $\xi$ and $s$, and are independent of $N$. We infer from Theorem EL that there exists a positive real number $\eta^{\prime}$ such that

$$
N \ll\left(\varepsilon\left(q_{N}\right)\right)^{-s-5} \cdot \log \left(\varepsilon^{-1}\left(q_{N}\right)\right) \leq\left(\varepsilon\left(q_{N}\right)\right)^{-s-5-\eta / 2} \leq\left(\log _{m+1} q_{N}\right)^{1-\eta^{\prime}},
$$

for $N$ sufficiently large. Furthermore, it follows from (5.2) that

$$
\log _{m+1} q_{N} \ll N .
$$

The combination of (5.3) and (5.4) gives a contradiction.

This proves the theorem.

\section{Proofs of Theorems 3.1 and 3.2 and their corollaries}

Proof of Theorem 3.1. Under the assumptions of Theorem 3.1, we suppose that there are infinitely many solutions to (3.1), but that (3.2) does not hold. Consequently, by extracting suitably a subsequence from the set of primitive solutions to (3.1), there exist a real number $C$ and an infinite sequence of primitive integer $(n+1)$-tuples $\mathbf{x}_{j}=\left(q_{j}, p_{1, j}, \ldots, p_{n, j}\right)$ such that, for every $j \geq 1$, the integer $q_{j}$ is positive,

$$
q_{j} \cdot\left|q_{j} \xi_{1}-p_{1, j}\right| \cdots\left|q_{j} \xi_{n}-p_{n, j}\right| \leq q_{j}^{-\varepsilon\left(q_{j}\right)},
$$

and

$$
H_{j}^{2} \leq H_{j+1} \leq H_{j}^{C},
$$

where we have set $H_{j}:=\max \left\{q_{j},\left|p_{1, j}\right|, \ldots\left|p_{n, j}\right|\right\}$.

We will ultimately derive a contradiction. Throughout the present proof, $D$ denotes the degree of the number field generated by $\xi_{1}, \ldots, \xi_{n}$. The constants implied by $\ll, \gg$ depend at most on $C, n, \xi_{1}, \ldots, \xi_{n}$.

Let $c_{1}$ be a positive real number to be fixed later on. Let $N_{0}$ be a (large) integer such that, for any $q \geq q_{N_{0}}$, we have

$$
\varepsilon(q) \geq c_{1}(\log \log q)^{-1 /(2 n+6)} .
$$


Let $N \geq N_{0}$ be an even integer and set

$$
\varepsilon=\varepsilon\left(q_{N}\right) .
$$

Consider the $n+1$ linearly independent linear forms

$$
L_{0}(\mathbf{X})=X_{0}, \quad L_{1}(\mathbf{X})=\xi_{1} X_{0}-X_{1}, \ldots, L_{n}(\mathbf{X})=\xi_{n} X_{0}-X_{n} .
$$

Let $H$ be an upper bound for the heights of the linear forms $L_{i}, i=0, \ldots, n$. Our assumption implies that, for $N$ large enough, the equation

$$
\prod_{i=0}^{n}\left|L_{i}(\mathbf{x})\right|<H(\mathbf{x})^{-n-\varepsilon / 2}
$$

has at least $1+N / 2$ primitive (integer) solutions $\mathbf{x}=\left(x_{0}, x_{1}, \ldots, x_{n}\right)$ with

$$
\max \left\{\left|x_{0}\right|, \ldots,\left|x_{n}\right|\right\}>(n+1)^{4(n+1) / \varepsilon}+H,
$$

namely the $(n+1)$-tuples $\mathbf{x}_{j}$, with $N / 2 \leq j \leq N$. By Theorem ES, these $1+N / 2$ tuples are contained in the union of at most $c_{2} \varepsilon^{-2 n-6}$ rational proper subspaces of $\mathbb{Q}^{n+1}$, where $c_{2}=c_{2}(D, n)$ depends only on $D$ and $n$.

Furthermore, we infer from (6.1) that

$$
\log \log q_{N} \leq N \log \left(\mathrm{CH}_{1}\right)
$$

thus, by our choice of $\varepsilon$,

$$
c_{2} \varepsilon^{-2 n-6} \leq c_{1}^{-2 n-6} c_{2}\left(\log \log q_{N}\right) \leq c_{1}^{-2 n-6} c_{2} N \log \left(C H_{1}\right) .
$$

Choose $c_{1}$ large enough in order to satisfy

$$
2 c_{1}^{-2 n-6} c_{2} n(n+1) D \log \left(C H_{1}\right)<1 .
$$

Then, the primitive $(n+1)$-tuples $\mathbf{x}_{j}$ with $N / 2 \leq j \leq N$ are contained in a union of less than $N /(2 n(n+1) D)$ proper rational subspaces of $\mathbb{Q}^{n+1}$. Consequently, if $N$ is large enough, then there exists a proper rational subspace of $\mathbb{Q}^{n+1}$ that contains at least $t:=n(n+1) D$ of these tuples, namely the tuples $\mathbf{x}_{j_{1}}, \ldots, \mathbf{x}_{j_{t}}$, where $N / 2 \leq j_{1}<\ldots<j_{t} \leq N$. Set $M=n D$. For $k=1, \ldots, n+1$, let $V_{k}$ be the rational subspace spanned by $\mathbf{x}_{j_{1}}, \ldots, \mathbf{x}_{j_{k M}}$. Since $\operatorname{dim} V_{1} \geq 1$ and $\operatorname{dim} V_{n+1} \leq n$, there exists $k$ such that $1 \leq k \leq n$ and $V_{k}=V_{k+1}$. Consequently, there exists an integer $(n+1)$-tuple $\left(z_{0}, z_{1}, \ldots, z_{n}\right)$ such that

$$
z_{0} q_{j_{h}}+z_{1} p_{1, j_{h}}+\ldots+z_{n} p_{n, j_{h}}=0, \quad \text { for } h=1, \ldots,(k+1) M,
$$

and

$$
Z:=\max \left\{\left|z_{0}\right|, \ldots,\left|z_{n}\right|\right\} \ll H_{j_{k M}}^{n}
$$


Since, by assumption, we have

$$
\left|q_{j} \xi_{i}-p_{i, j}\right| \leq 1, \quad \text { for } 1 \leq i \leq n \text { and } 1 \leq j \leq N
$$

we deduce from (6.2) that

$$
\begin{aligned}
n Z & \geq\left|z_{1}\left(q_{j_{h}} \xi_{1}-p_{1, j_{h}}\right)+\ldots+z_{n}\left(q_{j_{h}} \xi_{n}-p_{n, j_{h}}\right)\right| \\
& =q_{j_{h}}\left|z_{0}+z_{1} \xi_{1}+\ldots+z_{n} \xi_{n}\right|, \quad \text { for } h=k M+1, \ldots,(k+1) M .
\end{aligned}
$$

Since $\xi_{1}, \ldots, \xi_{n}$ are algebraic, we infer from Lemma 4.1 and (6.3) that

$$
\left|z_{0}+z_{1} \xi_{1}+\ldots+z_{n} \xi_{n}\right| \gg Z^{-D} \gg H_{j_{k M}}^{-n D} .
$$

It follows from (6.3), (6.4) and (6.5) that $q_{j_{(k+1) M}}$ is bounded in terms of $H_{j_{k M}}$, namely that we have

$$
H_{j_{(k+1) M}} \ll q_{j_{(k+1) M}} \ll H_{j_{k M}}^{n(D+1)} .
$$

However, the gap condition (6.1) yields

$$
H_{j_{(k+1) M}} \geq H_{j_{k M}}^{2^{M}}
$$

Our choice $M=n D$ implies that (6.6) and (6.7) do not hold simultaneously when $H_{j_{k M}}$ is large enough, that is, when $N$ is large enough. We have reached a contradiction. Thus, the sequence $\left(\log H_{j+1} / \log H_{j}\right)_{j \geq 1}$ cannot be bounded. This establishes the theorem, since $q_{j} \ll H_{j} \ll q_{j}$ for $j \geq 1$.

For the proof of Theorem 3.2, we need an auxiliary result.

Lemma 6.1. Let $n$ be a positive integer and let $\xi_{1}, \ldots, \xi_{n}$ be real algebraic numbers such that $1, \xi_{1}, \ldots, \xi_{n}$ are linearly independent over the rationals. Let $\varepsilon$ be a positive real number. Assume that there are $M$ primitive integer $(n+1)$-tuples $\mathbf{p}_{j}:=\left(p_{0, j}, p_{1, j}, \ldots, p_{n, j}\right)$ such that

$$
\begin{gathered}
\left|p_{0, j}+p_{1, j} \xi_{1}+\ldots+p_{n, j} \xi_{n}\right|<H_{j}^{-n-\varepsilon}, \\
H_{1} \geq \max \left\{H\left(\xi_{1}\right), \ldots, H\left(\xi_{n}\right)\right\},
\end{gathered}
$$

and

$$
H_{j}^{2} \leq H_{j+1}, \quad \text { for } j=1, \ldots, M
$$

where we have set

$$
H_{j}:=\max \left\{\left|p_{0, j}\right|,\left|p_{1, j}\right|, \ldots,\left|p_{n, j}\right|\right\}, \quad \text { for } j=1, \ldots, M .
$$

Then, $M$ is bounded effectively in terms of $\varepsilon$ and of the degree of the field generated by $\xi_{1}, \ldots, \xi_{n}$. 
Proof. The proof goes by induction on $n$. The lemma is a direct consequence of Theorem EL if $n=1$. Let $n \geq 2$ be an integer, and assume that the lemma holds for every positive integer less than $n$.

The integers $c_{1}, c_{2}, \ldots$ occurring below depend at most on $\varepsilon$ and on the degree of the field generated by $\xi_{1}, \ldots, \xi_{n}$.

Consider the $n+1$ linearly independent linear forms

$$
L_{0}(\mathbf{X})=X_{0}+\xi_{1} X_{1}+\ldots+\xi_{n} X_{n}, \quad L_{1}(\mathbf{X})=X_{1}, \ldots, L_{n}(\mathbf{X})=X_{n} .
$$

Our assumption implies that the equation

$$
\prod_{i=0}^{n}\left|L_{i}(\mathbf{x})\right|<H(\mathbf{x})^{-n-\varepsilon}
$$

has at least $M$ primitive solutions.

We infer from Theorem ES that, for $M \geq c_{1}$, the $(n+1)$-tuples $\mathbf{p}_{j}$ with $M / 2 \leq$ $j \leq M$ are lying in the union of at most $c_{2}$ proper subspaces of $\mathbb{Q}^{n+1}$. Let $y_{0} X_{0}+$ $\ldots+y_{n} X_{n}=0$ be one of these subspaces, and assume that it contains the $(n+1)$ tuples $\mathbf{p}_{j_{1}}, \mathbf{p}_{j_{2}}, \ldots, \mathbf{p}_{j_{t}}$, where $M / 2 \leq j_{1}<j_{2}<\ldots<j_{t} \leq M$ and $t=2(n+1) c_{3}$. Set $L=2 c_{3}$. For $k=1, \ldots, n+1$, let $V_{k}$ be the rational subspace spanned by $\mathbf{p}_{j_{1}}, \ldots, \mathbf{p}_{j_{k L}}$. Since $\operatorname{dim} V_{1} \geq 1$ and $\operatorname{dim} V_{n+1} \leq n$, there exists $k$ such that $1 \leq k \leq$ $n$ and $V_{k}=V_{k+1}$. Consequently, there exists an integer $(n+1)$-tuple $\left(z_{0}, z_{1}, \ldots, z_{n}\right)$ such that

$$
z_{0} p_{0, j_{h}}+z_{1} p_{1, j_{h}}+\ldots+z_{n} p_{n, j_{h}}=0, \quad \text { for } h=1, \ldots,(k+1) L,
$$

and

$$
Z:=\max \left\{\left|z_{0}\right|, \ldots,\left|z_{n}\right|\right\} \leq c_{4} H_{j_{k L}}^{n} .
$$

Let $h$ be an integer such that $k L \leq h \leq(k+1) L$. By (6.8) and

$$
\left|p_{0, j_{h}}+p_{1, j_{h}} \xi_{1}+\ldots+p_{n, j_{h}} \xi_{n}\right|<H_{j_{h}}^{-n-\varepsilon},
$$

we get that

$$
\left|p_{0, j_{h}}\left(z_{0} \xi_{n}-z_{n}\right)+p_{1, j_{h}}\left(z_{1} \xi_{n}-z_{n} \xi_{1}\right)+\ldots+p_{n-1, j_{h}}\left(z_{n-1} \xi_{n}-z_{n} \xi_{n-1}\right)\right|<Z \cdot H_{j_{h}}^{-n-\varepsilon}
$$

Without any loss of generality, we may assume that $\left(z_{0}, z_{n}\right) \neq(0,0)$. Set

$$
\zeta_{i}=\frac{z_{i} \xi_{n}-z_{n} \xi_{i}}{z_{0} \xi_{n}-z_{n}}, \quad \text { for } i=1, \ldots, n-1
$$

Observe that, for $c_{3}$ large enough, we have

$$
Z \leq H_{j_{(k+1 / 2) L}}^{1 / 2} \quad \text { and } \quad H_{j_{(k+1 / 2) L}} \geq \max \left\{H\left(\zeta_{1}\right), \ldots, H\left(\zeta_{n-1}\right)\right\}
$$


For $h=k L+L / 2, \ldots,(k+1) L$, the $n$-tuple $\left(p_{0, j_{h}}, p_{1, j_{h}}, \ldots, p_{n-1, j_{h}}\right)$ has no reason to be primitive. However, it follows from (6.8) and from the primitivity of $\mathbf{p}_{j_{h}}$ that the greatest common divisor of $p_{0, j_{h}}, \ldots, p_{n-1, j_{h}}$ cannot exceed $Z$. Since we have

$$
\begin{gathered}
0<\left|p_{0, j_{h}}+p_{1, j_{h}} \zeta_{1}+\ldots+p_{n-1, j_{h}} \zeta_{n-1}\right|<H_{j_{h}}^{-(n-1)-1 / 2} \\
\quad \text { for } h=k L+L / 2, \ldots,(k+1) L,
\end{gathered}
$$

it follows from our hypothesis of induction that $L \leq c_{5}$. Consequently, $M \leq c_{6}$, as asserted.

We are now in position to establish Theorem 3.2.

Proof of Theorem 3.2. We assume that the sequence of solutions to (3.3) is infinite and does not satisfy the gap condition (3.4). Consequently, by extracting suitably a subsequence from the set of primitive solutions to (3.3), there exist a real number $C$ and an infinite sequence of primitive integer $(n+1)$-tuples $\mathbf{p}_{j}:=$ $\left(p_{0, j}, p_{1, j}, \ldots, p_{n, j}\right)$ such that

$$
\left|p_{0, j}+p_{1, j} \xi_{1}+\ldots+p_{n, j} \xi_{n}\right| \leq H_{j}^{-n-\varepsilon\left(H_{j}\right)}
$$

and

$$
H_{j}^{2} \leq H_{j+1} \leq H_{j}^{C}, \quad \text { for } j \geq 1
$$

where we have set

$$
H_{j}:=\max \left\{\left|p_{0, j}\right|,\left|p_{1, j}\right|, \ldots,\left|p_{n, j}\right|\right\}, \quad \text { for } j \geq 1 .
$$

Throughout the present proof, $D$ denotes the degree of the number field generated by $\xi_{1}, \ldots, \xi_{n}$.

Let $c_{1}$ be a positive real number to be fixed later on. Let $N_{0}$ be a (large) integer such that, for any $H \geq H_{N_{0}}$, we have

$$
\varepsilon(H) \geq c_{1}(\log \log H)^{-1 /(2 n+6)} .
$$

Let $N \geq N_{0}$ be an even integer, and set

$$
\varepsilon=\varepsilon\left(H_{N}\right) .
$$

Consider the $n+1$ linearly independent linear forms

$$
L_{0}(\mathbf{X})=X_{0}+\xi_{1} X_{1}+\ldots+\xi_{n} X_{n}, \quad L_{1}(\mathbf{X})=X_{1}, \ldots, L_{n}(\mathbf{X})=X_{n} .
$$

Let $H$ be an upper bound for the heights of the linear forms $L_{i}, i=0, \ldots, n$. Our assumption implies that, for $N$ large enough, the equation

$$
\prod_{i=0}^{n}\left|L_{i}(\mathbf{x})\right|<H(\mathbf{x})^{-n-\varepsilon / 2}
$$


has at least $1+N / 2$ primitive (integer) solutions $\mathbf{x}=\left(x_{0}, x_{1}, \ldots, x_{n}\right)$ with

$$
\max \left\{\left|x_{0}\right|, \ldots,\left|x_{n}\right|\right\}>(n+1)^{4(n+1) / \varepsilon}+H,
$$

namely the $(n+1)$-tuples $\mathbf{p}_{j}$, with $N / 2 \leq j \leq N$. By Theorem ES, these $1+N / 2$ tuples are contained in the union of at most $c_{2} \varepsilon^{-2 n-6}$ rational proper subspaces of $\mathbb{Q}^{n+1}$, where $c_{2}$ depends only on $n$ and $D$.

Furthermore, we infer from (6.9) that

$$
\log \log H_{N} \leq N \log \left(\mathrm{CH}_{1}\right),
$$

thus

$$
c_{2} \varepsilon^{-2 n-6}=c_{1}^{-2 n-6} c_{2}\left(\log \log q_{N}\right) \leq c_{1}^{-2 n-6} c_{2} N \log \left(\mathrm{CH}_{1}\right) .
$$

Let $M$ be an even integer to be fixed later on. Choose $c_{1}$ large enough in order to satisfy

$$
2 c_{1}^{-2 n-6} c_{2}(n+1) M \log \left(C_{1}\right)<1 .
$$

Then, the primitive $(n+1)$-tuples $\mathbf{p}_{j}$ with $N / 2 \leq j \leq N$ are contained in a union of less than $N /(2(n+1) M)$ proper subspaces and, for $N$ large enough, at least one of these subspaces contains (at least) $M(n+1)$ of the tuples $\mathbf{p}_{j}$ with $N / 2 \leq j \leq N$. We proceed exactly as in the proof of Theorem 3.1 and we get that there exists an integer $(n+1)$-tuple $\left(z_{0}, z_{1}, \ldots, z_{n}\right)$ such that

$$
z_{0} p_{0, j_{h}}+z_{1} p_{1, j_{h}}+\ldots+z_{n} p_{n, j_{h}}=0, \quad \text { for } h=1, \ldots,(k+1) M,
$$

and

$$
Z:=\max \left\{\left|z_{0}\right|, \ldots,\left|z_{n}\right|\right\} \leq c_{3} H_{j_{k M}}^{n},
$$

for some $c_{3}$ depending only on $n$. By (6.10) and

$$
\left|p_{0, j_{h}}+p_{1, j_{h}} \xi_{1}+\ldots+p_{n, j_{h}} \xi_{n}\right| \leq H_{j_{h}}^{-n-\varepsilon},
$$

we get that

$\left|p_{0, j_{h}}\left(z_{0} \xi_{n}-z_{n}\right)+p_{1, j_{h}}\left(z_{1} \xi_{n}-z_{n} \xi_{1}\right)+\ldots+p_{n-1, j_{h}}\left(z_{n-1} \xi_{n}-z_{n} \xi_{n-1}\right)\right| \leq Z \cdot H_{j_{h}}^{-n-\varepsilon}$.

Without any loss of generality, we may assume that $\left(z_{0}, z_{n}\right) \neq(0,0)$. Set

$$
\zeta_{i}=\frac{z_{i} \xi_{n}-\xi_{i} z_{n}}{z_{0} \xi_{n}-z_{n}}, \quad \text { for } i=1, \ldots, n-1,
$$

and select $M$ sufficiently large in order that

$$
Z, H\left(\zeta_{1}\right), \ldots, H\left(\zeta_{n-1}\right) \leq H_{j_{k M+M / 2}}^{1 / 2} .
$$

Then, we get

$$
\begin{aligned}
& 0<\left|p_{0, j_{h}}+p_{1, j_{h}} \zeta_{1}+\ldots+p_{n-1, j_{h}} \zeta_{n-1}\right|<H_{j_{h}}^{-(n-1)-1 / 2}, \\
& \quad \text { for } h=k M+M / 2, \ldots,(k+1) M .
\end{aligned}
$$

Selecting $M$ sufficiently large in terms of $D$, we have reached a contradiction with Lemma 6.1. This establishes the theorem. 
Proofs of Corollaries 3.3 and 3.4. Applying Theorem 3.2 with $\xi_{i}=\xi^{i}$, for $i=$ $1, \ldots, n$, we get Corollary 3.3. Furthermore, under the assumption of Corollary 3.4, if we denote by $P_{j}(X)$ the minimal defining polynomial of $\alpha_{j}$ over the integers, then it is easily seen that

$$
\left|P_{j}(\xi)\right|<H\left(P_{j}\right)^{-n-\varepsilon\left(H\left(P_{j}\right)\right) / 2}
$$

holds for any sufficiently large $j$. By Corollary 3.3, we get (3.6), that is, (3.8), by definition of the height of an algebraic number.

\section{Fractional parts of powers of rational numbers}

Let $p$ and $q$ be coprime integers with $p>q \geq 2$. Let $\varepsilon$ be a positive real number. Applying a theorem of Ridout [23], Mahler [20] proved that

$$
\left\|\left(\frac{p}{q}\right)^{n}\right\| \geq 2^{-\varepsilon n}
$$

holds for every sufficiently large integer $n$. Here, as above, $\|\cdot\|$ denotes the distance to the nearest integer.

The application of the Cugiani-Mahler theorem yields further results on the fractional parts $\left\|(p / q)^{n}\right\|$, as pointed out by Mahler in his monograph [21, Theorem 2, page 176]; see also [22, Théorème 2]). However, in both works, there is an extra assumption on the rational $p / q$, namely that $p$ is prime.

In this Section, we show that our Theorem 2.4 above allows us to remove this assumption and to obtain a result comparable to that of Mahler and Mignotte.

Theorem 7.1. Let $p$ and $q$ be coprime integers with $p>q \geq 2$. Let $s$ be the number of distinct prime divisors of pq. Let $m$ be a non-negative integer. Let $\delta$ be a positive real number such that $\delta<1 /(s+5)$. If there exists a strictly increasing sequence $n_{1}<n_{2}<\ldots$ of positive integers such that

$$
\left\|\left(\frac{p}{q}\right)^{n_{j}}\right\| \leq \exp \left\{-\frac{n_{j}}{\left(\log _{m+1} n_{j}\right)^{\delta}}\right\}, \quad \text { for } j \geq 1,
$$

then

$$
\limsup _{j \rightarrow+\infty} \frac{\log _{m} n_{j+1}}{\log _{m} n_{j}}=+\infty
$$

Proof. Throughout this proof, the constants implied by $\ll$ and $\gg$ depend at most on $p$ and $q$. For $n \geq 1$, let $g_{n}$ be the nearest integer to $(p / q)^{n}$. Let $d_{n}$ be the greatest common divisor of $p^{n}$ and $g_{n} q^{n}$, and define

$$
P_{n}=\frac{p^{n}}{d_{n}}, \quad Q_{n}=\frac{g_{n}}{d_{n}} q^{n} .
$$


Let $S$ be the set of prime divisors of $p q$. For a positive integer $x$, we write $|x|_{S}$ for the product of the $|x|_{\ell}$ over all the prime numbers $\ell$ in $S$. Following Mahler's proof, we have

$$
\begin{gathered}
\left|P_{n} Q_{n}\right|_{S}=P_{n}^{-1} \cdot\left|Q_{n}\right|_{S} \leq P_{n}^{-1} \cdot q^{-n} \ll Q_{n}^{-1-(\log q) /(\log p)}, \\
g_{n} \gg Q_{n}^{1-(\log q) /(\log p)}, \quad q^{n} \leq Q_{n} \ll p^{n} .
\end{gathered}
$$

From our assumption, for $j$ sufficiently large, we have

$$
\begin{aligned}
& \left|1-\frac{P_{n_{j}}}{Q_{n_{j}}}\right| \cdot\left|P_{n_{j}} Q_{n_{j}}\right|_{S}=\frac{1}{g_{n_{j}}}\left|\left(\frac{p}{q}\right)^{n_{j}}-g_{n_{j}}\right| \cdot\left|P_{n_{j}} Q_{n_{j}}\right|_{S} \\
& \leq \frac{1}{g_{n_{j}}} \exp \left\{-\frac{n_{j}}{\left(\log _{m+1} n_{j}\right)^{\delta}}\right\} \cdot\left|P_{n_{j}} Q_{n_{j}}\right| S \\
& \ll Q_{n_{j}}^{-2-c\left(\log _{m+2} Q_{n_{j}}\right)^{-\delta}},
\end{aligned}
$$

for some positive constant $c$. Applying Theorem 2.4 with $\xi=1$, we get at once Theorem 7.1.

The results of Mahler and Mignotte, valid only when $p$ is prime, do not depend on the number of prime divisors of $q$. To achieve this, these authors apply instead of Theorem 2.4 a version of the Cugiani-Mahler theorem in which (2.4) is replaced by a system of inequalities. However, we were unable to get Theorem 7.1 in its full generality with this tool, and we needed a version of the Cugiani-Mahler theorem with a product, as in the left-hand side of (2.4).

\section{Final remarks}

An important application of the method developed in the present paper is concerned with the block complexity of irrational, algebraic numbers. Let $b \geq 2$ be an integer and $\xi$ be an irrational, algebraic number with $0<\xi<1$. There exists a unique infinite sequence $\mathbf{a}=\left(a_{j}\right)_{j \geq 1}$ of integers from $\{0,1, \ldots, b-1\}$, called the $b$-ary expansion of $\xi$, such that

$$
\xi=\sum_{j \geq 1} \frac{a_{j}}{b^{j}} .
$$

A natural way to measure the complexity of $\xi$ (in base $b$ ) is to count the number of distinct blocks of given length in the infinite word a. To this end, for an infinite word $\mathbf{w}$ on the alphabet $\{0,1, \ldots, b-1\}$ and for a positive integer $n$, we let $p(n, \mathbf{w})$ denote the number of distinct blocks of $n$ letters occurring in w. Furthermore, we set $p(n, \xi, b)=p(n, \mathbf{a})$ with a as above. In 1997 Ferenczi and Mauduit [17] applied a non-Archimedean extension of Roth's theorem established by Ridout [23] to show (see also [4]) that

$$
\lim _{n \rightarrow+\infty}(p(n, \xi, b)-n)=+\infty
$$


Then, a new combinatorial transcendence criterion proved with the help of the Schmidt Subspace theorem (precisely, its non-Archimedean extension) by Adamczewski, Bugeaud, and Luca [3] enabled Adamczewski and Bugeaud [2] to establish that

$$
\lim _{n \rightarrow+\infty} \frac{p(n, \xi, b)}{n}=+\infty .
$$

By using the Quantitative Subspace theorem (precisely, its non-Archimedean extension from [15]) in a similar way as in the proofs of Theorems 3.1 and 3.2, it is possible to complement (8.1) by establishing that, for any positive real number $\varepsilon$, we have

$$
\limsup _{n \rightarrow+\infty} \frac{p(n, \xi, b)}{n(\log n)^{-\varepsilon+1 /(4 \omega(b)+15)}}=+\infty,
$$

where $\omega(b)$ denotes the number of distinct prime factors of $b$. However, it turns out that, by means of a more careful use of the Quantitative Subspace theorem, the dependence on $b$ in (8.2) can be removed. Namely, it is proved in [9] that (8.2) holds with $1 /(4 \omega(b)+15)$ replaced by $1 / 11$.

Likewise, we may improve the lower bound for the complexity of irrational $p$-adic algebraic numbers established in [2, Theorem 1B].

The approach followed in the present paper can be used to get new, explicit examples of transcendental continued fractions, in the same spirit as in [1]. This will be the subject of a forthcoming note.

To conclude, we wish to emphasize an important open question:

To find an upper bound for the number of solutions to (1.6).

At present, this question has been solved only when the exponent $-n-1-\varepsilon$ is strictly smaller than $-2 n$, see $[14,19]$.

\section{References}

[1] B. Adamczewski and Y. Bugeaud, On the complexity of algebraic numbers, II. Continued fractions, Acta Math. 195 (2005), 1-20.

[2] B. AdAMCZEWSKI and Y. BUGEAUD, On the complexity of algebraic numbers I. Expansions in integer bases, Ann. of Math. 165 (2007), 547-565.

[3] B. Adamczewsi, Y. Bugeaud et F. LuCA, Sur la complexité des nombres algébriques, C. R. Acad. Sci. Paris 339 (2004), 11-14.

[4] J.-P. Allouche, Nouveaux résultats de transcendance de réels à développements non aléatoire, Gaz. Math. 84 (2000), 19-34.

[5] V. BERESNEVICH, On approximation of real numbers by real algebraic numbers, Acta Arith. 90 (1999), 97-112.

[6] V. I. BERNIK, On the best approximation of zero by values of integral polynomials, Acta Arith. 53 (1989), 17-28 (in Russian).

[7] E. Bombieri and W. GubleR, "Heights in Diophantine Geometry", New mathematical monographs 4, Cambridge University Press, 2006.

[8] E. BOMbIERI and A. J. VAN DER POORTEn, Some quantitative results related to Roth's theorem, J. Aust. Math. Soc. 45 (1988), 233-248. 
[9] Y. Bugeaud and J.-H. EverTse, On two notions of complexity of algebraic numbers, preprint available at http: / / arxiv.org/pdf / 0709.1560.

[10] M. Cugiani, Sull'approssimazione di numeri algebrici mediante razionali, In: "Collectanea Mathematica", Pubblicazioni dell'Istituto di Matematica dell'Università di Milano 169, C. Tanburini (ed.), Milano, 1958, pages 5.

[11] M. Cugiani, Sulla approssimabilità dei numeri algebrici mediante numeri razionali, Ann. Mat. Pura Appl. 48 (1959), 135-145.

[12] M. Cugiani, Sull'approssimabilità di un numero algebrico mediante numeri algebrici di un corpo assegnato, Boll. Unione Mat. Ital. 14 (1959), 151-162.

[13] H. DAVENPORT and K. F. Roth, Rational approximations to algebraic numbers, Mathematika 2 (1955), 160-167.

[14] J.-H. EVERTSE, The number of algebraic numbers of given degree approximating a given algebraic number. In: "Analytic Number Theory" (Kyoto, 1996), London Math. Soc. Lecture Note Ser. 247, Cambridge Univ. Press, Cambridge, 1997, 53-83.

[15] J.-H. EVERTSE and H.P. SCHLICKEWEI, A quantitative version of the Absolute Subspace Theorem, J. Reine Angew. Math. 548 (2002), 21-127.

[16] P. FATOU, Sur l'approximation des incommensurables et des séries trigonométriques, C. R. Acad. Sci. Paris 139 (1904), 1019-1021.

[17] S. FERENCZI and CH. MAUdUIT, Transcendence of numbers with a low complexity expansion, J. Number Theory 67 (1997), 146-161.

[18] J. H. GRACE, The classification of rational approximations, Proc. London Math. Soc. 17 (1918), 247-258.

[19] H. LOCHER, On the number of good approximations of algebraic numbers by algebraic numbers of bounded degree, Acta Arith. 89 (1999), 97-122.

[20] K. MAHLER, On the fractional parts of the powers of a rational number, II, Mathematika 4 (1957), 122-124.

[21] K. Mahler, "Lectures on Diophantine Approximation, Part 1: g-Adic Numbers and Roth's Theorem", University of Notre Dame, Ann Arbor, 1961.

[22] M. Mignotte, Une généralisation d'un théorème de Cugiani-Mahler, Acta Arith. 22 (1972), 57-67.

[23] D. RIDout, Rational approximations to algebraic numbers, Mathematika 4 (1957), 125131.

[24] G. RodriQuez, Approssimabilità di irrazionali p-adici mediante numeri razionali, Ist. Lombardo Accad. Sci. Lett. Rend. A 98 (1964), 691-708.

[25] G. RodriQuez, Approssimabilità di irrazionali p-adici mediante numeri razionali. II, Boll. Unione Mat. Ital. 20 (1965), 232-244.

[26] K. F. Rотн, Rational approximations to algebraic numbers, Mathematika 2 (1955), 1-20; corrigendum, 168.

[27] W. M. SCHMIDT, Über simultane Approximation algebraischer Zahlen durch Rationale, Acta Math. 114 (1965) 159-206.

[28] W. M. SCHMIDT, On simultaneous approximations of two algebraic numbers by rationals, Acta Math. 119 (1967), 27-50.

[29] W. M. SCHMIDT, Simultaneous approximations to algebraic numbers by rationals, Acta Math. 125 (1970), 189-201.

[30] W. M. Schmidt, Norm form equations, Ann. of Math. 96 (1972), 526-551.

[31] W. M. SchmidT, "Diophantine Approximation", Lecture Notes in Mathematics, Vol. 785, Springer, 1980.

[32] W. M. Schmidt, The subspace theorem in Diophantine approximation, Compositio Math. 69 (1989), 121-173.

[33] K. B. Stolarsky, "Algebraic numbers and Diophantine Approximation", Pure and Applied Mathematics, Vol. 26, Marcel Dekker, Inc., New York, 1974. 
[34] M. WALDSChmidt, "Diophantine Approximation on Linear Algebraic Groups, Transcendence Properties of the Exponential Function in Several Variables", Grundlehren der Mathematischen Wissenschaften, Vol. 326, Springer-Verlag, Berlin, 2000.

Université Louis Pasteur U. F. R. de mathématiques 7 , rue René Descartes 67084 Strasbourg, Cedex, France bugeaud@math.u-strasbg.fr 